\title{
An investigation into the effects of dietary supplementation of coconut oil on blood lipids and anthropometric measurements in healthy adults
}

\author{
J. Webb, I. Alaunyte and F. Amirabdollahian \\ School of Health Sciences, Liverpool Hope University, Liverpool, L16 9JD
}

Coconut oil is a natural food product containing relatively high amounts of MCFAs. A number of studies that have been conducted to assess the effects of coconut oil supplementation on health benefits produced equivocal results ${ }^{(1,2)}$. Medium-chain fatty acids (MCFAs) have recently received a lot of attention due to their substantial metabolic difference when compared with long-chain fatty acids (LCFAs) ${ }^{(3)}$. The proposed benefits associated with consumption of MCFAs products include weight control, glucose and lipid metabolism ${ }^{(1)}$. The aim of the present study was to investigate the effects of incorporating virgin coconut oil (VCO) into the habitual diet on anthropometric measurements and blood lipid profiles in healthy adults.

After obtaining ethical approval, mixed gender participants ( $n 35$, aged $18-50)$ were recruited and assigned to a control $(n$ 14, no dietary change) or intervention group ( $n$ 21, replacement of usual cooking oil with VCO for 4 weeks). The anthropometric measurements and fasting capillary blood lipid profile markers were obtained at baseline and post intervention. Energy and nutrient intakes were assessed using a 3-day food diary and analysed using dietary assessment software Microdiet. Statistical analysis was conducted by repeated measures ANOVA using SPSS (v 22) and statistical significance was set at $\mathrm{P}<0.05$.

Baseline anthropometric and blood lipid markers were not significantly different between participants in control and intervention groups showing the groups were matched. The dietary composition of the diets at baseline and post intervention was the same except for saturated fatty acids in intervention group due to the inclusion of VCO. The results showed that there were no significant changes in body weight, BMI, body fat $\%$, fat mass, fat free mass, waist and hip circumference between the groups. In addition to this, there were no significant changes in total cholesterol (TC), HDL and LDL values between the groups.

\begin{tabular}{|c|c|c|c|c|c|c|c|}
\hline \multirow[b]{2}{*}{ Parameter } & \multicolumn{2}{|c|}{ Control group $(n=14)$} & \multicolumn{2}{|c|}{$\begin{array}{l}\text { Virgin Coconut oil group } \\
(\mathrm{n}=21)\end{array}$} & \multirow[b]{2}{*}{ Time } & \multirow[b]{2}{*}{ Group } & \multirow[b]{2}{*}{ Group $\times$ Tim } \\
\hline & $\overline{\text { Mean }}$ & $\overline{\mathrm{SD}}$ & $\overline{\text { Mean }}$ & $\mathrm{SD}$ & & & \\
\hline \multicolumn{8}{|l|}{ Weight (kg) } \\
\hline Baseline & $64 \cdot 8$ & $13 \cdot 9$ & 67.4 & $14 \cdot 0$ & \multirow[t]{3}{*}{$P=0.429$} & \multirow[t]{3}{*}{$P=0.448$} & \multirow[t]{3}{*}{$P=0.448$} \\
\hline End & $64 \cdot 1$ & $13 \cdot 9$ & $67 \cdot 4$ & $13 \cdot 9$ & & & \\
\hline \multicolumn{5}{|c|}{ Waist circumference $(\mathrm{cm})$} & & & \\
\hline Baseline & $76 \cdot 3$ & 11.4 & $77 \cdot 6$ & $10 \cdot 2$ & \multirow[t]{3}{*}{$P=0.547$} & \multirow[t]{3}{*}{$P=0.064$} & \multirow[t]{3}{*}{$P=0.064$} \\
\hline End & $77 \cdot 4$ & $12 \cdot 4$ & $75 \cdot 5$ & $10 \cdot 4$ & & & \\
\hline \multicolumn{5}{|c|}{ Waist to Hip ratio } & & & \\
\hline Baseline & 0.77 & $0 \cdot 07$ & $0 \cdot 81$ & 0.79 & \multirow[t]{3}{*}{$P=0.784$} & \multirow[t]{3}{*}{$\mathrm{P}=0 \cdot 145$} & \multirow[t]{3}{*}{$\mathrm{P}=0.145$} \\
\hline End & 0.79 & $0 \cdot 80$ & 0.79 & $0 \cdot 60$ & & & \\
\hline \multicolumn{5}{|c|}{ TC (mmol/L) } & & & \\
\hline Baseline & $4 \cdot 13$ & $0 \cdot 80$ & $4 \cdot 07$ & 0.47 & \multirow[t]{2}{*}{$P=0.324$} & \multirow[t]{2}{*}{$\mathrm{P}=0.619$} & \multirow[t]{2}{*}{$\mathrm{P}=0.054$} \\
\hline End & 3.88 & 0.67 & $4 \cdot 15$ & 0.66 & & & \\
\hline
\end{tabular}

To conclude, the present study found that in contrast to previous research ${ }^{(2)}$, short-term incorporation of coconut oil into the habitual diet showed no effects on anthropometric and blood lipid profile in free-living healthy adults.

1. Schwab Niskanen, Maliranta et al. (1995) J Nutr 125, 466-473.

2. Temme Mensink and Hornstra (1997) J Lipid Res 38, 1746-54.

3. Marten Pfeuffer and Schrezenmeir (2006) Int Dairy J 16, 1374-1382. 